\title{
Effects of dietary fat on gut microbiota and faecal metabolites, and their relationship with cardiometabolic risk factors: a 6-month randomised controlled-feeding trial
}

\author{
Yi Wan, ${ }^{1}$ Fenglei Wang, ${ }^{1,2}$ Jihong Yuan, ${ }^{3}$ Jie Li, ${ }^{3}$ Dandan Jiang, ${ }^{3}$ Jingjing Zhang, ${ }^{4}$ \\ Hao Li, ${ }^{1}$ Ruoyi Wang, ${ }^{1,2}$ Jun Tang, ${ }^{1}$ Tao Huang, ${ }^{5}$ Jusheng Zheng, ${ }^{6}$ Andrew J Sinclair, ${ }^{7}$ \\ Jim Mann, ${ }^{8}$ Duo Li ${ }^{1,9}$
}

Additional material for this paper is available online. To view these files, please visit the journal online (http://dx.doi.org/ 10.1136/gutjnl-2018-317609).

For numbered affiliations see end of article.

\section{Correspondence to}

Professor Duo Li, Institute of Nutrition and Health, Qingdao University, Qingdao 266071,

China; duoli@qdu.edu.cn

YW and FW contributed equally.

Received 17 September 2018 Revised 3 December 2018 Accepted 8 December 2018

Published Online First

19 February 2019

\section{ABSTRACT}

Objective To investigate whether diets differing in fat content alter the gut microbiota and faecal metabolomic profiles, and to determine their relationship with cardiometabolic risk factors in healthy adults whose diet is in a transition from a traditional low-fat diet to a diet high in fat and reduced in carbohydrate.

Methods In a 6-month randomised controlled-feeding trial, 217 healthy young adults (aged 18-35 years; body mass index $<28 \mathrm{~kg} / \mathrm{m}^{2} ; 52 \%$ women) who completed the whole trial were included. All the foods were provided during the intervention period. The three isocaloric diets were: a lower-fat diet (fat 20\% energy), a moderate-fat diet (fat 30\% energy) and a higher-fat diet (fat 40\% energy). The effects of the dietary interventions on the gut microbiota, faecal metabolomics and plasma inflammatory factors were investigated.

Results The lower-fat diet was associated with increased $\alpha$-diversity assessed by the Shannon index $(p=0.03)$, increased abundance of Blautia $(p=0.007)$ and Faecalibacterium $(p=0.04)$, whereas the higher-fat diet was associated with increased Alistipes $(p=0.04)$, Bacteroides $(p<0.001)$ and decreased Faecalibacterium $(p=0.04)$. The concentration of total short-chain fatty acids was significantly decreased in the higher-fat diet group in comparison with the other groups $(p<0.001)$. The cometabolites p-cresol and indole, known to be associated with host metabolic disorders, were decreased in the lower-fat diet group. In addition, the higher-fat diet was associated with faecal enrichment in arachidonic acid and the lipopolysaccharide biosynthesis pathway as well as elevated plasma proinflammatory factors after the intervention.

Conclusion Higher-fat consumption by healthy young adults whose diet is in a state of nutrition transition appeared to be associated with unfavourable changes in gut microbiota, faecal metabolomic profiles and plasma proinflammatory factors, which might confer adverse consequences for long-term health outcomes. Trial registration number NCT02355795; Results. employer(s)) 2019. No commercial re-use. See rights and permissions. Published by BMJ.

\section{To cite: Wan Y, Wang F,} Yuan J, et al. Gut

2019:68:1417-1429.

\section{INTRODUCTION}

The parallel increases in dietary carbohydrate and the prevalence of obesity and type 2 diabetes (T2D)

\section{Significance of this study}

What is already known on this subject?

- Rates of obesity and other cardiometabolic disorders in most developing countries have increased rapidly in parallel with a transition from the traditional lower-fat diet to a diet relatively high in fat but lower in carbohydrate.

- Gut microbiota dysbiosis has been shown to be associated with a high risk of obesity, type 2 diabetes and many other cardiometabolic diseases.

- Studies using rodent models suggest that a high-fat diet unbalances the gut microbiota and impairs the gut barrier, resulting in cardiometabolic diseases.

- Observational studies conducted in globally distinct human populations suggest that diet has a strong effect on the composition of gut microbiota and related faecal metabolomics, while evidence from dietary intervention studies suggests that the effect of diet on gut microbiota might be more modest.

in the United States and some other Western countries have led to the suggestion that this dietary change, resulting from an increase in carbohydrate consumption and a reduction in total fat intake, might be a determinant of cardiometabolic disorders. ${ }^{1-3}$ By contrast in China, the nutritional transition from the traditional low-fat, high-carbohydrate diet to a diet relatively higher in fat and lower in carbohydrate has been associated with a dramatic increase in the risk of obesity, T2D and cardiovascular diseases in the past 30 years. ${ }^{45} \mathrm{We}$ have previously shown in a randomised controlledfeeding trial that among healthy young adults, a lower-fat, higher-carbohydrate diet is likely to be associated with a lower risk of excessive weight gain and increase in waist circumference and a more favourable lipid profile than a higher-fat, lower-carbohydrate diet. ${ }^{6}$

There has been interest in the role of gut microbiota in the development of obesity and 
Significance of this study

What are the new findings?

- At the phylum level, the moderate-fat and higher-fat diets decreased the ratio of Firmicutes to Bacteroidetes after the intervention.

- At the genus level, the higher-fat diet decreased the abundance of Faecalibacterium, increased the abundance of Alistipes and Bacteroides, while the lower-fat diet increased the Faecalibacterium and Blautia abundance after the intervention.

- Concentration of faecal butyric acids was increased after the lower-fat diet intervention and decreased after the higher-fat diet intervention. The cometabolites $p$-cresol and indole were decreased after consumption of the lower-fat diet, while palmitic acid (C16:0), stearic acid (C18:0) and arachidonic acid were increased after the higher-fat diet consumption.

- Change in relative abundance of Blautia was negatively associated with the changes in serum total cholesterol, low-density lipoprotein cholesterol and non-high-density lipoprotein cholesterol, whereas the change in Bacteroides abundance was positively correlated with the changes in these three blood lipid markers.

- For circulating proinflammatory factors, the higher-fat diet was associated with increased plasma high sensitivity $C$-reactive protein and thromboxane $B_{2}$ relative to the lowerfat diet, while leukotriene $B_{4}$ and prostaglandin $E_{2}$ in the lower-fat diet were decreased the most among the three diet groups.

\section{How might it impact on clinical practice in the foreseeable future?}

- Our findings demonstrate that among healthy young adults whose diet is in a state of transition, a higher-fat diet had a notably unfavourable impact on gut microbial taxa, faecal metabolomic profiles and plasma proinflammatory biomarkers, whereas the lower-fat diet was associated with a more favourable profile of these biomarkers. These findings provide confirmatory evidence that nutritional guidelines in countries in a state of nutrition transition should advise against increasing intakes of dietary fat. The results might also have relevance in developed countries in which fat intake is already high.

cardiometabolic diseases. ${ }^{7}$ Rodent studies have suggested that the high-fat Western-type diet has a strong effect on the genetic composition and metabolic activity of gut microbiota. ${ }^{89}$ Evidence has shown that in humans gut microbiota diversity and richness are reduced when comparing such high-fat diets with more traditional diets with relatively higher proportions of carbohydrate. ${ }^{10}{ }^{11}$ Such diet-induced 'dysbiosis' in gut-associated microbial communities has been postulated as a major trigger of metabolic impairments associated with obesity. ${ }^{12}$ Much of the relevant existing literature on humans is based on observational studies and the relatively few short-term dietary intervention trials suggest that the effect of dietary change might be relatively modest. ${ }^{13-16}$ No information is available relating to healthy young people or to populations whose diet is in transition from that traditionally consumed to one associated with a high risk of cardiometabolic disease.

Thus in a 6-month randomised controlled-feeding trial among healthy young adults, we compared a number of attributes of gut microbiota and faecal metabolomic profiles as well as markers of inflammation for diets of differing proportions of fat and carbohydrate.

\section{METHODS}

\section{Study design and population}

This study investigated the gut microbiota, faecal metabolomic profiles response and their relationship with cardiometabolic risk factors in 217 healthy adults who had provided faecal samples in the Optimal Dietary Macronutrient Distribution in China trial. ${ }^{6}$ The trial was conducted at People's Liberation Army General Hospital in north China and Zhejiang University in south China and was approved by the ethics committees. Each participant provided written informed consent and received no financial compensation or gifts. The trial was registered at ClinicalTrials.gov, number NCT02355795.

The primary aim of the trial was to determine whether the traditional lower-fat diet was more effective than a higher-fat diet at weight control among a healthy young population whose diet was in a state of nutrition transition. The study design, method and primary results have been described elsewhere. ${ }^{617}$ In brief, the trial involved screening 1145 potentially eligible adults in two centres from January 2015. The main inclusion criteria included body mass index $<28 \mathrm{~kg} / \mathrm{m}^{2}$, the lower cut-off point according to the Chinese obesity criteria. Key exclusion criteria were blood pressure $\geq 140 \mathrm{~mm} \mathrm{Hg}$ systolic or $\geq 90 \mathrm{~mm}$ $\mathrm{Hg}$ diastolic, total cholesterol (TC) $\geq 6.19 \mathrm{mmol} / \mathrm{L}$, low-density lipoprotein cholesterol (LDL-C) $\geq 4.12 \mathrm{mmol} / \mathrm{L}$, fasting triglyceride $\geq 2.25 \mathrm{mmol} / \mathrm{L}$ and fasting glucose $\geq 6.11 \mathrm{mmol} / \mathrm{L}$. Finally, 307 eligible participants were randomised (1:1:1) to one of three diets with differing proportions of dietary fat, stratified by study centre, age, sex and body mass index using a computer-generated random number list. After 6 months of controlledfeeding intervention, 245 participants $(79.8 \%)$ completed the whole trial. Among them, a total of 217 participants who provided faecal samples at baseline and the end of the trial were included in the $16 \mathrm{~S}$ rRNA sequencing. Additionally, 120 participants ( $\mathrm{n}=40$ in each diet group) with enough faecal samples after $16 \mathrm{~S}$ rRNA sequencing were further included in the faecal metabolomic analysis.

\section{Study diets and intervention}

The three diets were isocaloric, the primary distinguishing feature being their fat and carbohydrate content. We achieved the required macronutrient distribution in the three diet groups by replacing a proportion of energy derived from carbohydrate (mainly white rice and wheat flour, the most consumed carbohydrate sources in Asia) with fats (mainly soybean oil, the most widely used edible oil in Asia). Otherwise, the menus for the three diet groups were almost identical. The intervention diets were a lower-fat diet (fat $20 \%$ and carbohydrate $66 \%$ energy, corresponding to the macronutrient distribution 30 years ago during which obesity was rare in China); a moderate-fat diet (fat $30 \%$ and carbohydrate 56\% energy, comparable with current macronutrient intake in China and also the upper limit of fat intake recommended by the Chinese Nutrition Society) and a higher-fat diet (fat $40 \%$ and carbohydrate $46 \%$ energy, approximating the current consumption of Chinese residents of some megacities). Protein provided 14\% energy in all three diets. In order to avoid an additional dietary variable, intake of dietary fibre on all the three diets was maintained at the baseline level of consumption, around $14 \mathrm{~g} /$ day. Details of the study diets are provided in the online supplementary table 1 . Participants were 
provided with all of their food and most beverages throughout the intervention. During the controlled-feeding period, they were asked to complete a daily diary in which they recorded whether they had eaten all the study foods and provided a list of non-study foods eaten.

\section{Blood specimen collection and assessment of proinflammatory factors}

Blood samples were collected by venepuncture after a 12-hour fast. Plasma and serum were centrifuged from the blood samples and immediately stored at $-80^{\circ} \mathrm{C}$ for further analysis. Serum concentrations of clinical biomarkers, including lipid profiles, glucose and insulin, were measured as described previously. ${ }^{6}$ Plasma proinflammatory factor concentrations, including interleukin (IL) $-1 \beta$, IL-6, IL-8, tumour necrosis factor- $\alpha$ (TNF- $\alpha$ ), prostaglandin $\mathrm{E}_{2}\left(\mathrm{PGE}_{2}\right)$, thromboxane $\mathrm{B}_{2}\left(\mathrm{TXB}_{2}\right)$ and leukotriene $\mathrm{B}_{4}\left(\mathrm{LTB}_{4}\right)$, were measured using ELISA kits (Cayman Chemical Co, Ann Arbour, Michigan, USA and MultiSciences Biotech Co, Hangzhou, China). In addition, high-sensitive C-reactive protein (hs-CRP) was measured by a latex-enhanced turbidimetric immunoassay method. All measurements were performed at the end of the study to minimise variability.

\section{$16 S$ rRNA sequencing and measurement of faecal metabolomics}

Faecal samples were collected at baseline and the end of the 6-month intervention. Each faecal sample was snap frozen in liquid nitrogen within minutes of donation and then kept at $-80^{\circ} \mathrm{C}$. DNA was extracted from stool samples $(200 \mathrm{mg})$ using QIAamp DNA Stool Mini Kit (Qiagen, Hilden, Germany) following the manufacturer's instructions, with additional homogenised steps in a bead beater (FastPrep, Thermo Electron Co, Boston, Massachusetts, USA). The V3-V4 hypervariable regions of the bacteria $16 \mathrm{~S}$ rRNA gene were amplified with barcode-indexed primers 338F (5'-ACTCCTACGGGAGGCAGCAG-3') and 806R (5'-GGACTACHVGGGTWTCTAAT-3'). The purified amplicons were pooled in equimolar concentration and further paired-end sequencing was performed using an Illumina Miseq instrument (Illumina, San Diego, California, USA). Raw sequencing data have been deposited in the National Center for Biotechnology Information Sequence Read Archive database with accession number PRJNA480547. Targeted identification and measurement of faecal metabolomics were performed based on a validated method..$^{18}$ Details of the $16 \mathrm{~S}$ rRNA sequence data preparation and analysis as well as the quantitative measurement of faecal metabolomics are available in online supplementary materials.

\section{Statistical analyses}

Descriptive statistics included crude comparison of demographic factors, clinical parameters and dietary intake among the three diet groups. Differences among diets for 6-month changes from baseline of proinflammatory markers were tested by analysis of variance or Kruskal-Wallis test based on whether the data were normally distributed. The $\alpha$-diversity indices evaluating gut microbial community richness (the Ace and Chao1 estimators) and community diversity (the Shannon estimator) were calculated using Mothur. ${ }^{19}$ Principal coordinate analysis (PCoA) based on Bray-Curtis distance and permutational multivariate analysis of variance (PERMANOVA) were performed to compare the global microbiota composition before and after intervention in each group at phylum, genus and operational taxonomic unit (OTU) levels, respectively. Enterotypes were determined using the abundance of genus and OTUs separately using the methods suggested by Arumugam et al ${ }^{20}$ In univariate analysis of gut microbiota and predicted Kyoto Encyclopaedia of Genes and Genomes (KEGG) biochemical pathways in each group, a paired t-test or a Wilcoxon matched-pairs test was adopted and $\mathrm{p}$ values were adjusted for multiple comparison using the Benjamini-Hochberg false discovery rate (FDR). Correlations between changes in cardiometabolic risk factors, including body weight, waist and lipid profiles selected based on the previous results, ${ }^{6}$ and changes in genus relative abundance, were calculated using Spearman's rank or Pearson's correlation depending on the distribution of the data.

Orthogonal projection to latent structure-discriminant analysis (OPLS-DA) with unit variance scaling was performed to examine the overall microbial metabolites distribution before and after intervention in each group. The qualities of all OPLS-DA models were assessed with $R^{2} X$ (the total variation being explained by the model) and $\mathrm{Q}^{2}$ (denoting the predictability of the model). The significance of the models was further validated by cross-validation analysis of variance (CV-ANOVA). Univariate analysis was also conducted with $\mathrm{p}$ values adjusted for multiple testing by the Benjamini-Hochberg approach. Correlations between changes in faecal metabolites and changes in genus relative abundance, as well as proinflammatory markers were calculated by Spearman's rank test or Pearson's correlation test, when appropriate, in the full cohort. P values for the correlations between changes in faecal metabolites and changes in genus relative abundance were corrected for multiple comparisons.

Analyses were also conducted to examine the difference in gut microbiota and faecal metabolomic profiles across groups. PCoA based on Bray-Curtis distance and PERMANOVA were performed to compare the global microbiota composition among the three groups at phylum, genus and OTU levels, at baseline and after intervention, respectively. OPLS-DA with unit variance scaling and CV-ANOVA were performed to examine the overall microbial metabolites distribution across the three groups at baseline and after intervention. For the significantly changed bacteria and faecal metabolites after FDR correction in each group, Wilcoxon test was further performed to compare the changes in values between the dietary groups.

OPLS-DA and CV-ANOVA were performed with the software SIMCA-P + version 14.0, and all other statistical analyses were performed in $\mathrm{R}$ version 3.4. The main $\mathrm{R}$ packages used were 'ape' and 'vegan' for PCoA and PERMANOVA analyses and 'ade4' and 'clusterSim' for enterotype analysis.

\section{RESULTS}

\section{Characteristics of study participants}

No significant differences in anthropometric measurements or clinical parameters were observed among the three groups at baseline $(n=217$, table 1). Mean carbohydrate intake of the three groups was 55\% energy at baseline and during the intervention period $67 \%$ energy in the lower-fat diet group, $57 \%$ energy in the moderate-fat diet group and 48\% energy in the higher-fat diet group (online supplementary figure 1 ). Mean dietary fat consumption of the three groups was $32 \%$ at baseline, which changed to around 20\%,30\% and 40\% energy in the lower-fat diet group, moderate-fat diet group and higher-fat diet group, respectively, during the intervention period. Protein intake remained stable, at around $13-14 \%$ energy. After the 6-month controlled-feeding intervention, all groups lost weight, the reduction in the lower-fat diet group being significantly greater than in the higher-fat groups. Similarly, reduction in waist circumference, TC, high-density 
Table 1 Baseline characteristics of the participants included in the gut microbiota study ( $n=217$ )

\begin{tabular}{|c|c|c|c|}
\hline Characteristics & Lower-fat diet $(n=73)$ & Moderate-fat diet $(n=73)$ & Higher-fat diet $(n=71)$ \\
\hline Age (years) & $23.3(3.4)$ & $23.6(4.0)$ & $23.4(4.1)$ \\
\hline Female & $38(52.1 \%)$ & $38(52.1 \%)$ & $38(53.5 \%)$ \\
\hline Northern area & $35(47.9 \%)$ & $38(52.1 \%)$ & $34(47.9 \%)$ \\
\hline Weight $(\mathrm{kg})$ & $60.2(10.8)$ & $59.3(9.6)$ & $61.7(10.4)$ \\
\hline Body mass index $\left(\mathrm{kg} / \mathrm{m}^{2}\right)$ & $21.7(2.6)$ & $21.7(2.6)$ & $21.9(2.6)$ \\
\hline Waist circumference $(\mathrm{cm})$ & $75.9(9.2)$ & $76.6(8.6)$ & $76.1(8.6)$ \\
\hline \multicolumn{4}{|l|}{ Blood pressure (mm Hg) } \\
\hline Systolic & $116.5(10.6)$ & $115.8(9.6)$ & $114.9(9.6)$ \\
\hline Diastolic & $69.8(6.9)$ & $69.9(6.3)$ & $70.9(7.1)$ \\
\hline \multicolumn{4}{|l|}{ Blood biomarkers } \\
\hline $\mathrm{TC}(\mathrm{mmol} / \mathrm{L})$ & $4.2(0.8)$ & $4.1(0.7)$ & $4.0(0.7)$ \\
\hline LDL-C (mmol/L) & $2.4(0.6)$ & $2.4(0.6)$ & $2.3(0.5)$ \\
\hline $\mathrm{HDL}-\mathrm{C}(\mathrm{mmol} / \mathrm{L})$ & $1.5(0.3)$ & $1.5(0.3)$ & $1.5(0.3)$ \\
\hline Non-HDL-C (mmol/L) & $2.7(0.7)$ & $2.6(0.7)$ & $2.6(0.5)$ \\
\hline Triglycerides (mmol/L) & $0.8(0.3)$ & $0.8(0.3)$ & $0.8(0.3)$ \\
\hline Apolipoprotein A1 (mmol/L) & $1.6(0.2)$ & $1.6(0.2)$ & $1.6(0.2)$ \\
\hline Apolipoprotein B (mmol/L) & $0.7(0.2)$ & $0.7(0.2)$ & $0.7(0.1)$ \\
\hline Glucose (mmol/L) & $4.2(0.5)$ & $4.1(0.4)$ & $4.2(0.5)$ \\
\hline Insulin (pmol/L) & $69.6(20.5)$ & $64.6(22.0)$ & $67.8(22.9)$ \\
\hline \multicolumn{4}{|l|}{ Dietary intakes } \\
\hline Energy (male, kcal/day) & $2080.9(117.9)$ & $2099.9(118.8)$ & $2087.5(105.2)$ \\
\hline Energy (female, kcal/day) & $1696.3(94.5)$ & $1709.6(75.8)$ & $1686.2(96.3)$ \\
\hline Total fat (\% TE) & $30.6(5.1)$ & $31.0(4.3)$ & $30.8(4.4)$ \\
\hline Carbohydrate (\% TE) & $55.4(4.8)$ & $55.4(4.0)$ & $54.9(4.4)$ \\
\hline Protein (\% TE) & $14.1(1.9)$ & $13.6(1.9)$ & $14.3(1.8)$ \\
\hline Fibre (g/day) & $13.5(5.4)$ & $12.9(5.7)$ & $13.0(5.5)$ \\
\hline Cholesterol (mg/day) & $392.1(354.1)$ & $396.6(318.6)$ & $388.3(339.3)$ \\
\hline Physical activity (kcal/kg/hour) & $3.3(1.7)$ & $3.4(2.1)$ & $3.0(1.6)$ \\
\hline
\end{tabular}

Data are mean (SD) or $\mathrm{n}(\%)$.

HDL-C, high-density lipoprotein cholesterol; LDL-C, low- density lipoprotein cholesterol; TC, total cholesterol; TE, total energy.

lipoprotein cholesterol (HDL-C), LDL-C and non-HDL-C for the lower-fat diet group were significantly greater than those observed in the higher-fat diet group. However, the change in ratio of TC to HDL-C among the groups did not differ significantly throughout the intervention period. Changes in anthropometric data and clinical parameters during the 6-month intervention among participants included in the gut microbiota study $(n=217)$ are presented in online supplementary figure 2 .

\section{Effects of dietary interventions on gut microbiota}

The microbial community richness indicated by Ace and Chao1 estimators showed no significant changes among the three groups, whereas the community diversity estimated by the Shannon index was significantly increased in the lower-fat diet group relative to the higher-fat diet group $(p=0.03)$ (figure $1 \mathrm{~A})$. No significant shift was observed on the three diets in the overall composition of the gut microbiota at phylum, genus and OTU levels as indicated by PERMANOVA analysis (figure 1B-D). Similarly, the enterotypes did not change significantly in any of the three groups (figure 2 and online supplementary figure 3 ).

We further performed PCoA and PERMANOVA analyses among groups to investigate the potential differences at baseline and month 6 . At phylum level, we observed no statistically significant differences among the three diet groups at baseline, but marginally significant differences at month $6(\mathrm{p}=0.08)$. However, at genus and OTU level, there were no significant differences among groups at baseline or month 6 (online supplementary figure 4). Univariate analysis at phylum level showed that the relative abundance of Bacteroidetes was significantly increased in the moderate-fat diet group after intervention $(p=0.005$ after FDR correction) (figure $3 \mathrm{~A}$ ). In the higher-fat diet group, the relative abundance of Firmicutes was decreased, while Bacteroidetes was increased after intervention $(\mathrm{p}<0.001$ after FDR correction) (figure $3 \mathrm{~A}$ ). The ratio of Firmicutes to Bacteroidetes was significantly decreased in the moderate-fat and higher-fat diet group after intervention $(p=0.004$ for moderate fat diet group and $\mathrm{p}<0.001$ for higher-fat diet group). Compared with the lower-fat diet, the higher-fat diet significantly increased the abundance of Bacteroidetes and decreased the abundance of the Firmicutes (all $p<0.01$, online supplementary figure 5). At genus level, the lower-fat diet resulted in a significant increase in relative abundance of Blautia $(\mathrm{p}=0.007)$ and Faecalibacterium $(p=0.04)$ after the intervention; while the higher-fat diet led to a reduction in Faecalibacterium $(\mathrm{p}=0.04)$ and an increase in Alistipes $(\mathrm{p}=0.04)$ and Bacteroides $(\mathrm{p}<0.001)$, after FDR correction for multiple testing (figure $3 \mathrm{~B}$ and online supplementary table 2). Compared with lower-fat diet, the higher-fat diet led to a significant decrease in Blautia and Faecalibacterium abundance, while there was a significant increase in the abundance of Alistipes and Bacteroides (all $\mathrm{p}<0.01$, online supplementary figure 5 ). The change in relative abundance of Blautia was negatively associated with the changes in serum TC $(r=-0.27, p<0.001)$, LDL-C $(\mathrm{r}=-0.26, \mathrm{p}<0.001)$ and non-HDL-C $(\mathrm{r}=-0.24$, $\mathrm{p}<0.001$ ), whereas the change in Bacteroides was positively 
A $\alpha$-diversity
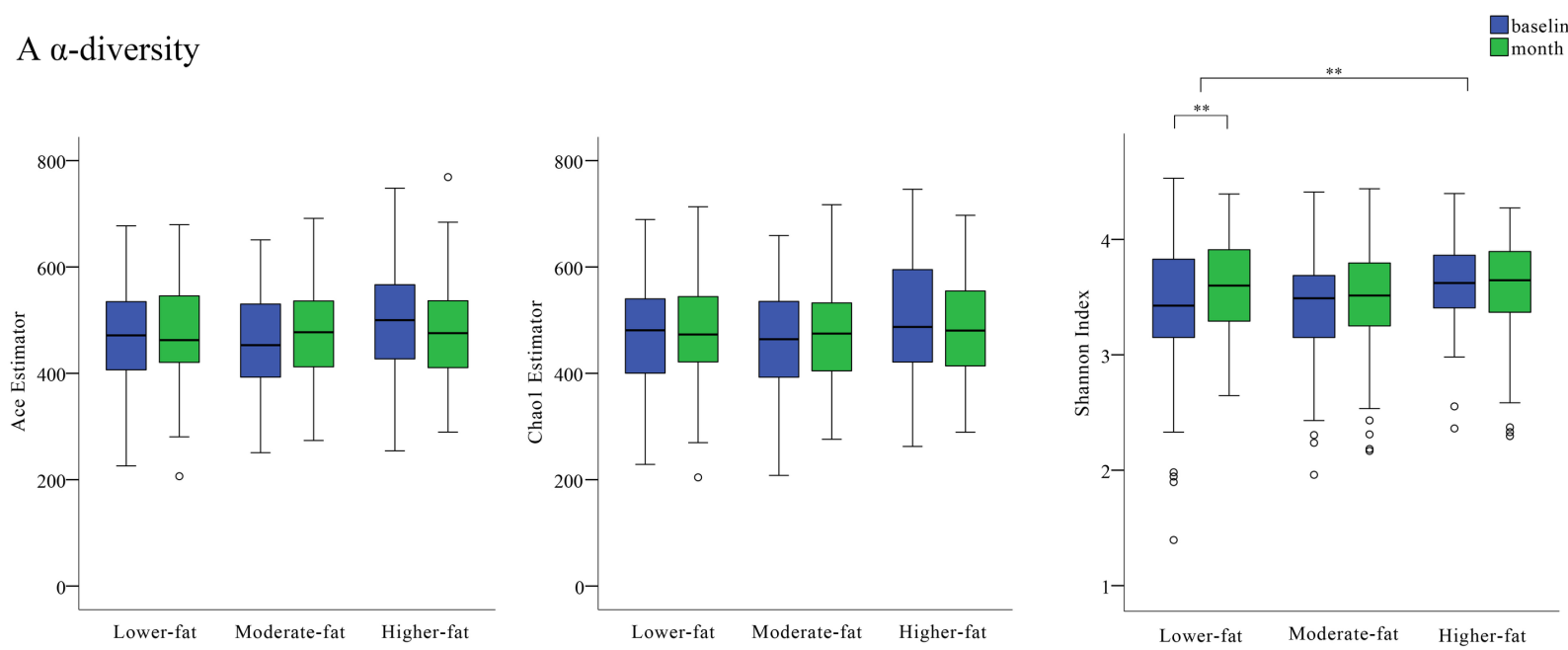

B Phylum
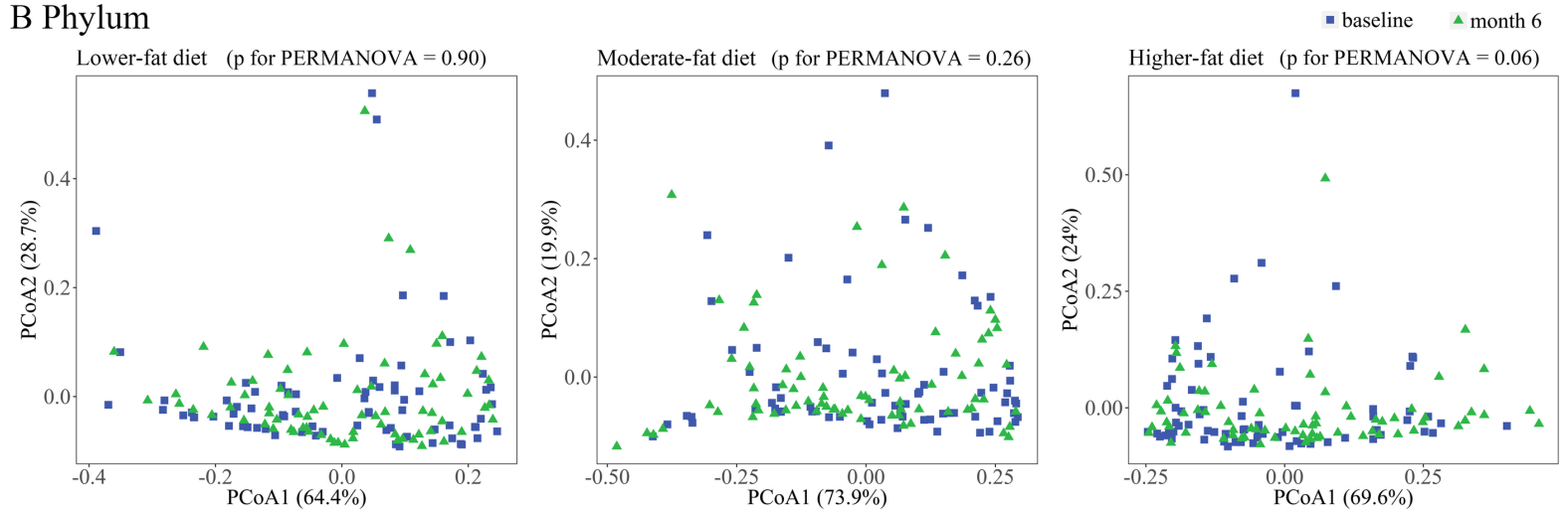

\section{Genus}
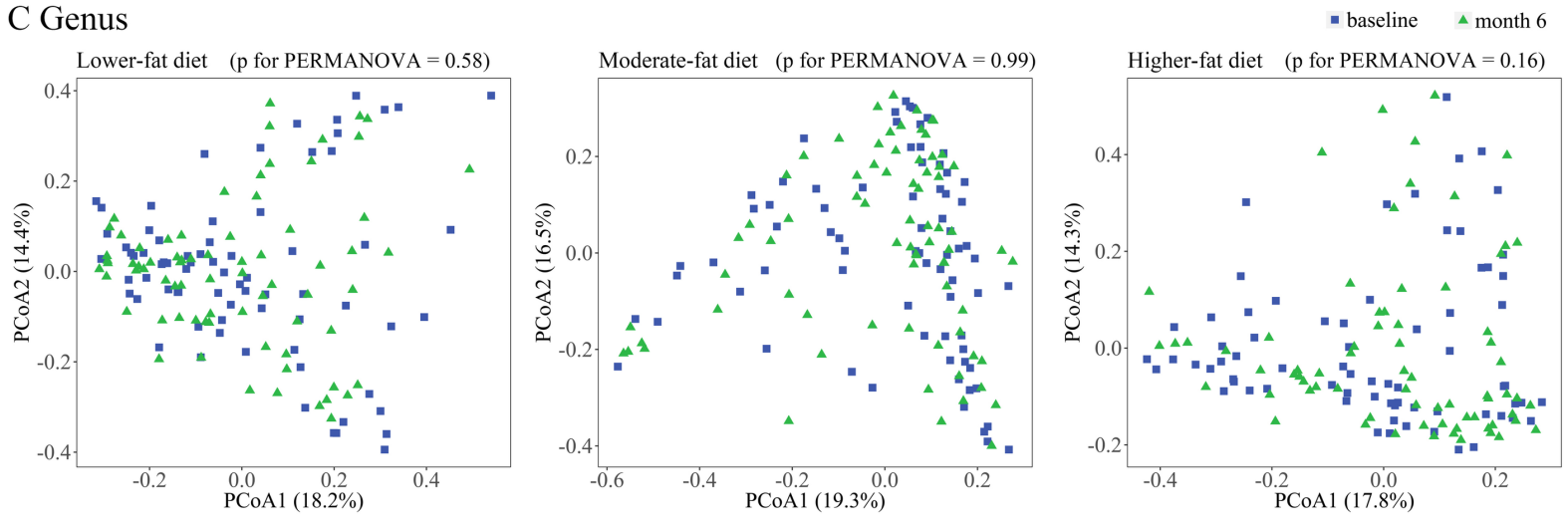

D OTU
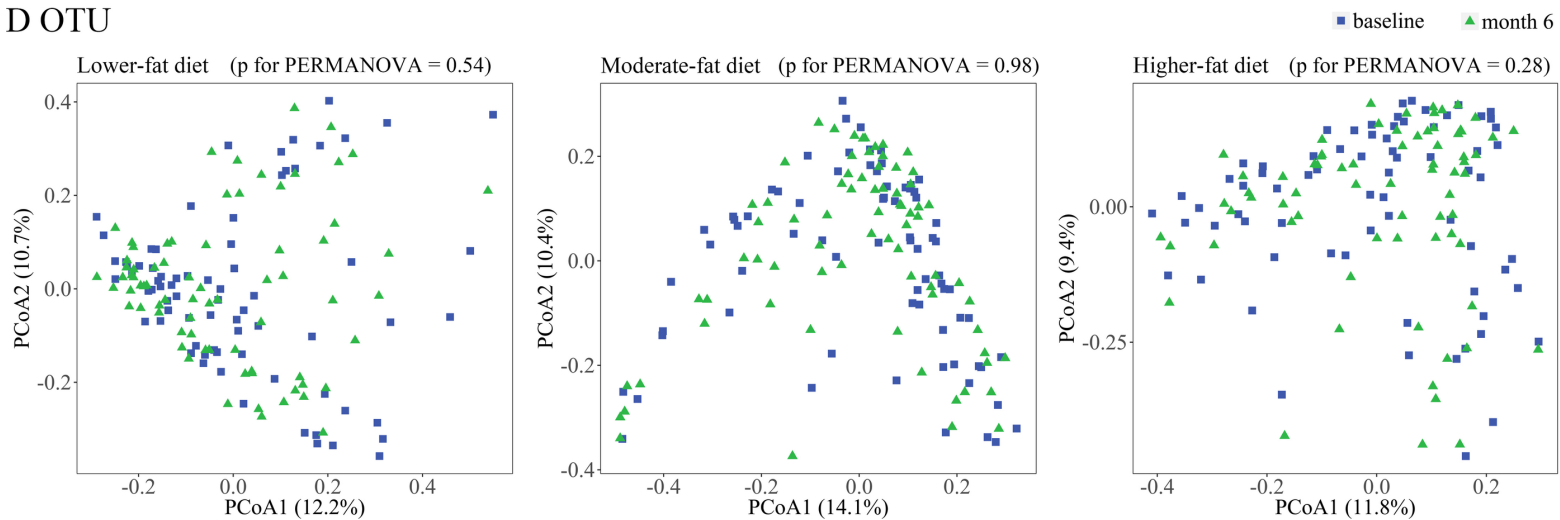

Figure 1 Changes of global gut microbiota after intervention in each group. (A) $\alpha$-Diversity at genus level estimated by Ace, Chao 1 and Shannon estimator. ${ }^{*} \mathrm{p}<0.05,{ }^{* *} \mathrm{p}<0.01$. (B) Principal coordinate analysis (PCoA) score plots based on Bray-Curtis distance at phylum level. (C) PCoA score plots based on Bray-Curtis distance at genus level. (D) PCoA score plots based on Bray-Cutis distance at operational taxonomic unit (OTU) level. 
A

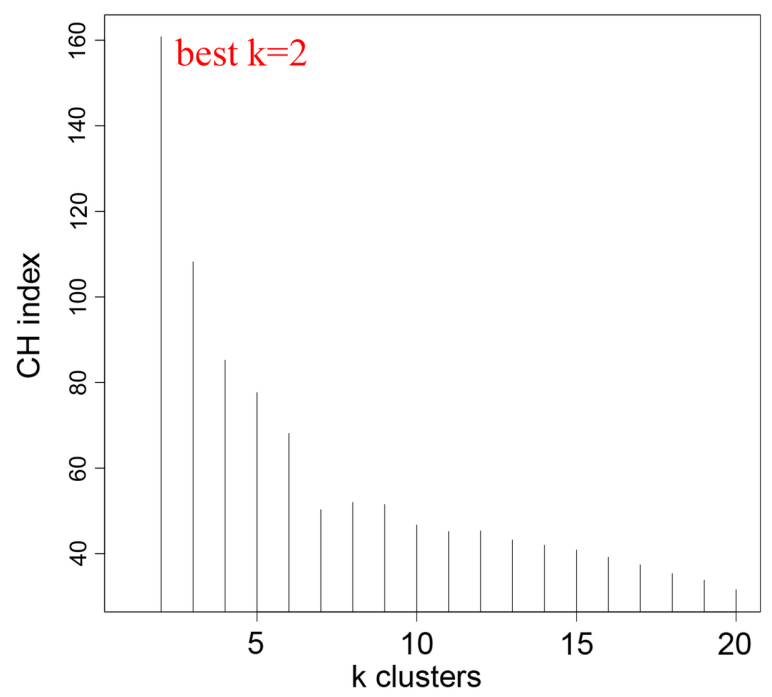

C

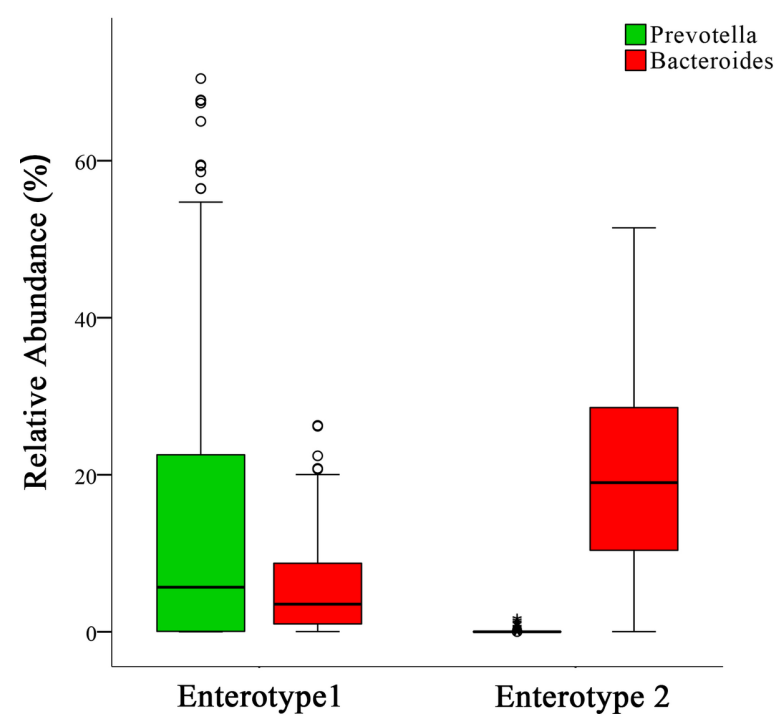

B

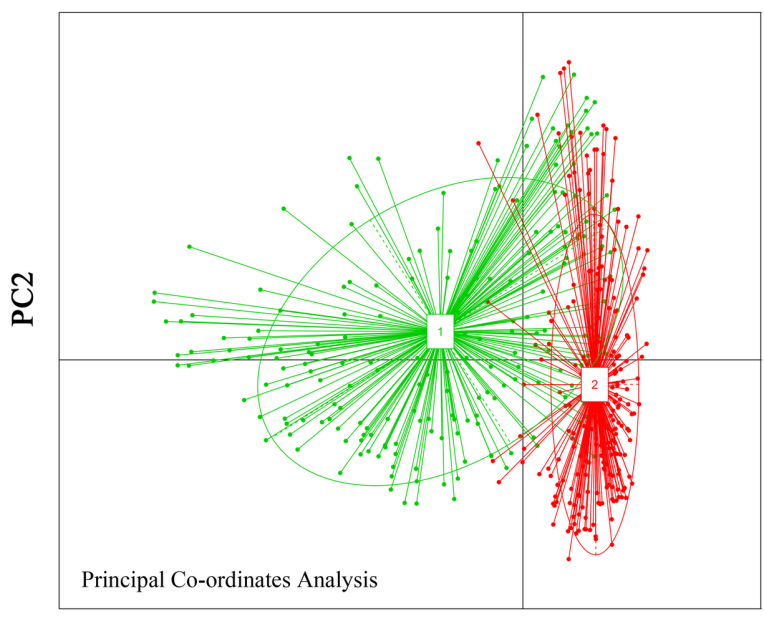

PC1

$\mathrm{D}$

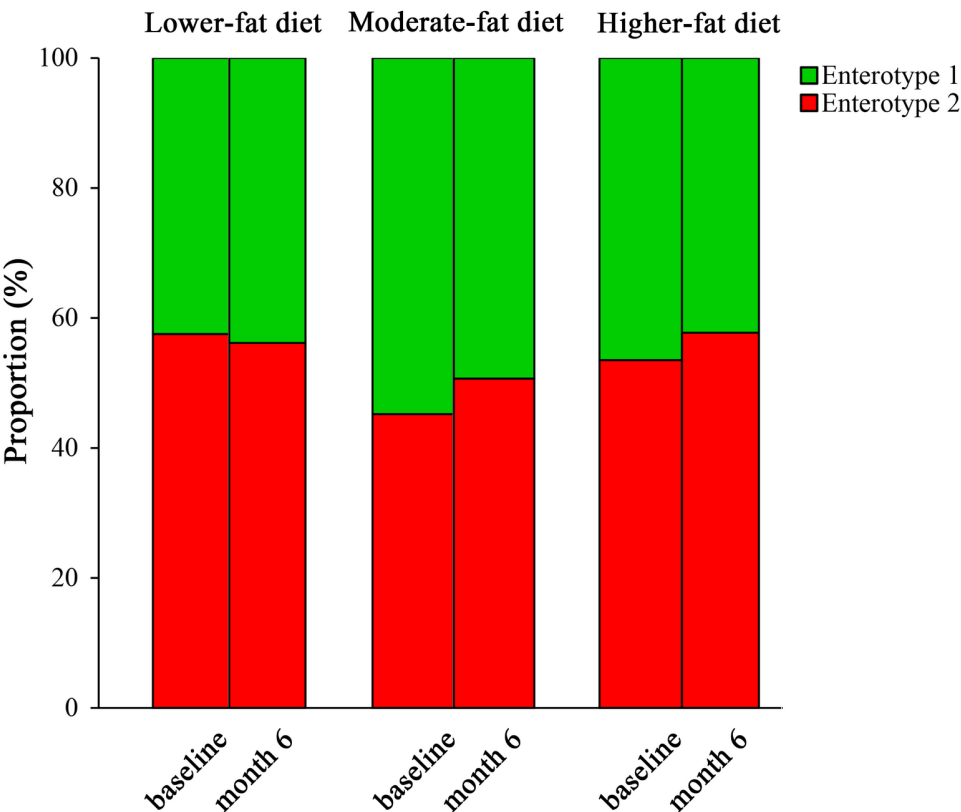

Figure 2 Clustering of gut microbiota into enterotypes at genus-level using Jensen-Shannon distance. (A) Two clusters exist most naturally in the dataset by the PAM method. The $x$-axis shows the cluster number, the $y$-axis shows the Calinski-Harabasz (CH) index. (B) Clustering of the first two principal components. (C) Relative abundance of bacterial taxa characteristic of each enterotype. Boxes represent the IQR and the line within represents the median. Whiskers denote the lowest and highest values within 1.5xIQR. (D) Proportions of enterotypes in each group before and after the intervention. No statistically significant changes were seen in each group before and after the intervention by $\chi^{2}$ tests.

correlated with the changes in these three blood lipid markers $(\mathrm{r}=0.44, \mathrm{p}<0.001$ for TC; $\mathrm{r}=0.34, \mathrm{p}<0.001$ for LDL-C and $\mathrm{r}=0.40, \mathrm{p}<0.001$ for non-HDL-C, respectively) (figure $3 \mathrm{C}$ ).

No significant changes in KEGG database biochemical pathways were seen in the lower-fat and moderate-fat groups after intervention (online supplementary table 3). Among the nine most responding pathways (FDR corrected $\mathrm{p}<0.10$ ) in the lower-fat group, lipopolysaccharide biosynthesis (ko00540) decreased to a notable degree (median fold change after intervention=0.75). In the higher-fat group, 29 pathways were identified as significantly responding to the higher-fat intervention after FDR correction (FDR corrected $\mathrm{p}<0.05$ ). Among these, pathways with notable changes (median fold change after intervention $\leq 0.80$ or $\geq 1.20$ ) were steroid hormone biosynthesis (ko00140), lysosome pathway (ko04142), arachidonic acid metabolism (ko00590) and lipopolysaccharide biosynthesis (ko00540). All four pathways were significantly increased in the higher-fat group.

\section{Effects of dietary interventions on faecal metabolomic profiles}

Significant shifts in the composition of faecal metabolites were observed after the lower-fat and higher-fat diets intervention $(p=0.02$ for lower-fat and $p=0.01$ for higher-fat diet) (figure 4A). Furthermore, no statistically significant differences were seen among groups at baseline (online supplementary figure 6). However, we observed significant differences among groups at month $6(p=0.002$, online supplementary figure 6). 
A
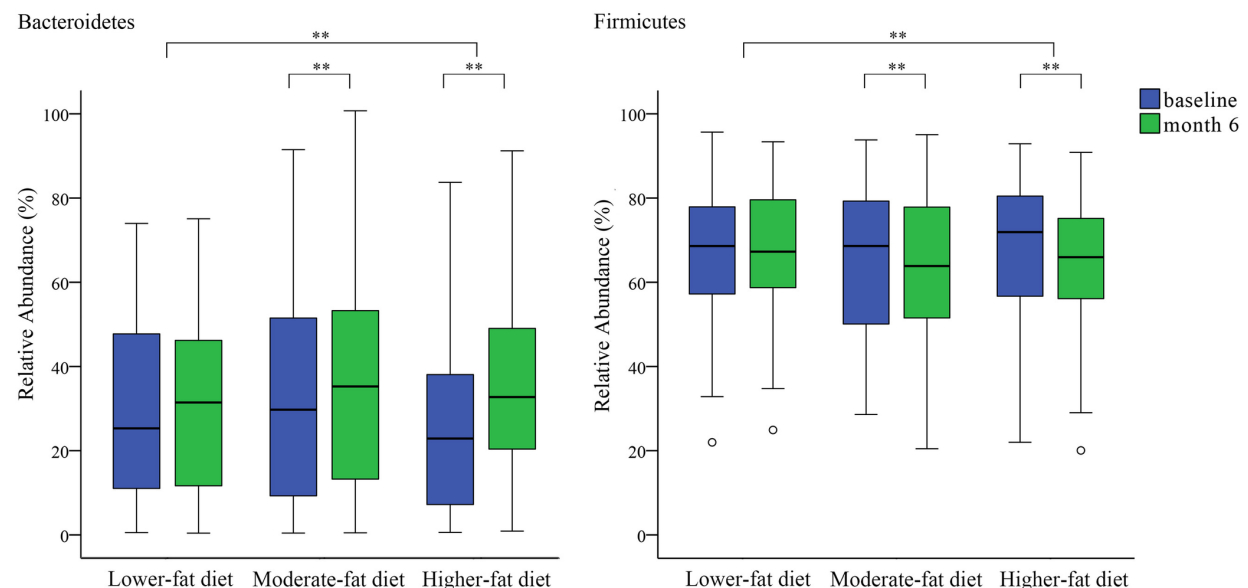

B

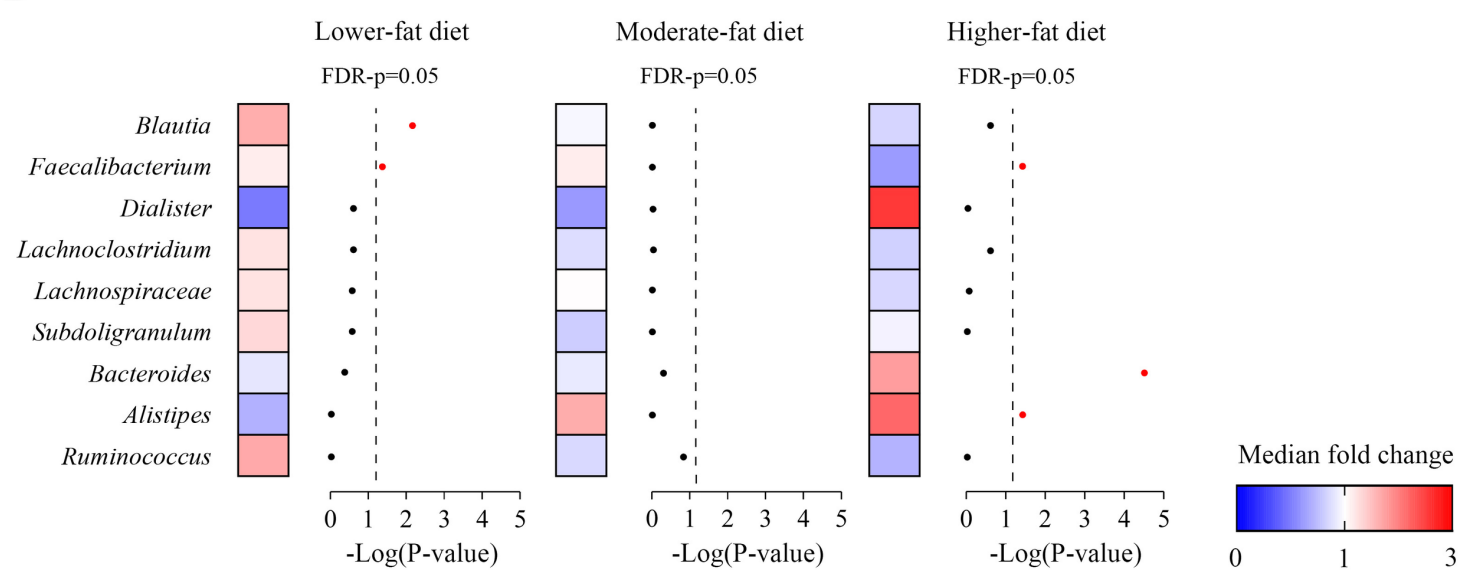

C
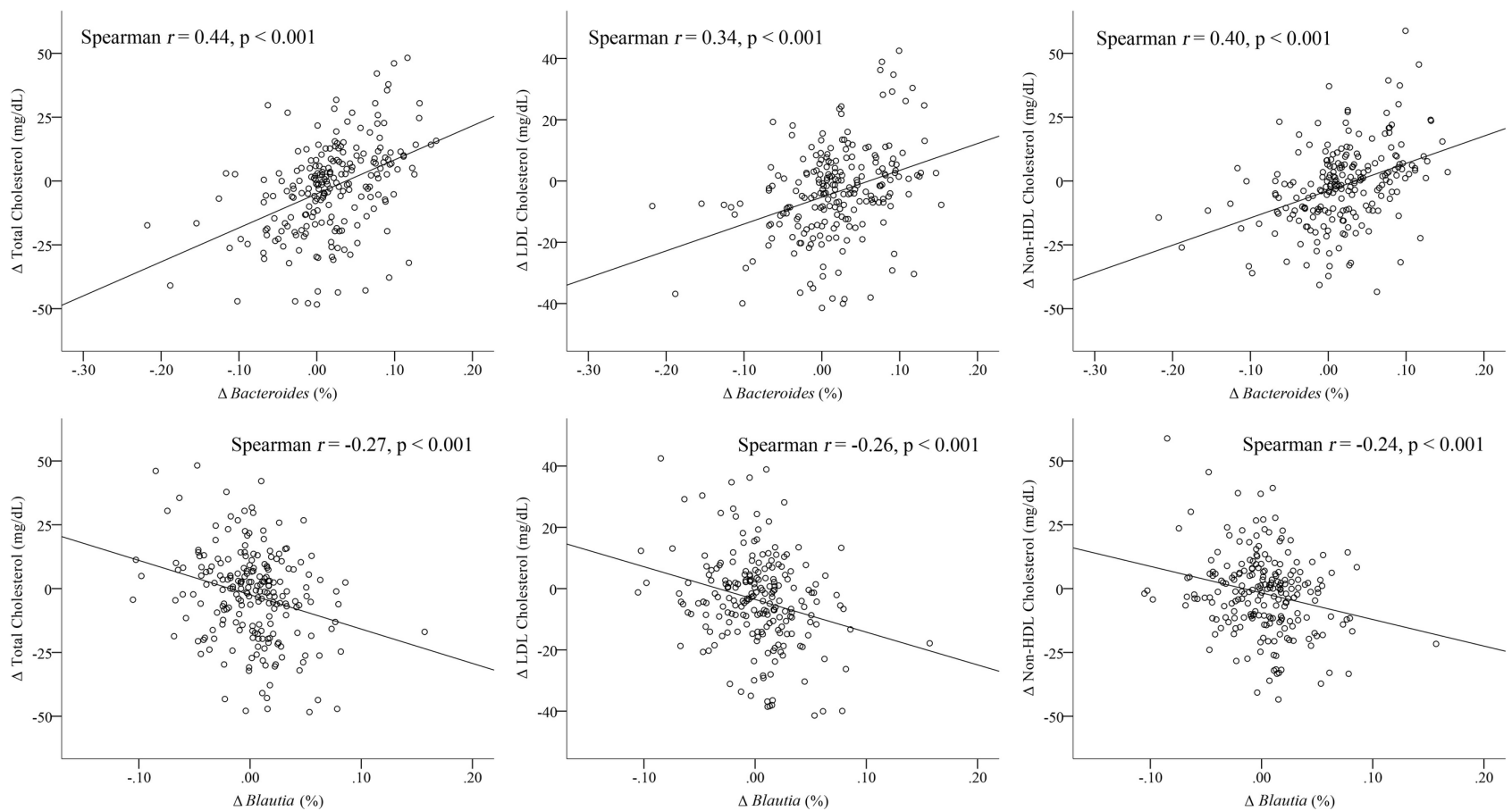

Figure 3 Analyses based on individual genera. (A) Changes of relative abundance of individual phylum before and after diet intervention. (B) Heatmap of the median fold changes in relative abundance of individual genera before and after the intervention. (C) Significant associations between changes in lipid profiles and changes in genus abundance as measured by the Spearman's correlations. ${ }^{*} \mathrm{p}<0.01$. FDR, false discovery rate. 
A

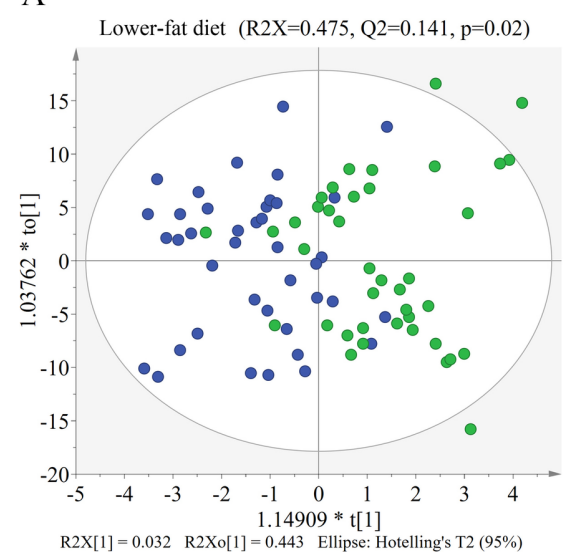

B

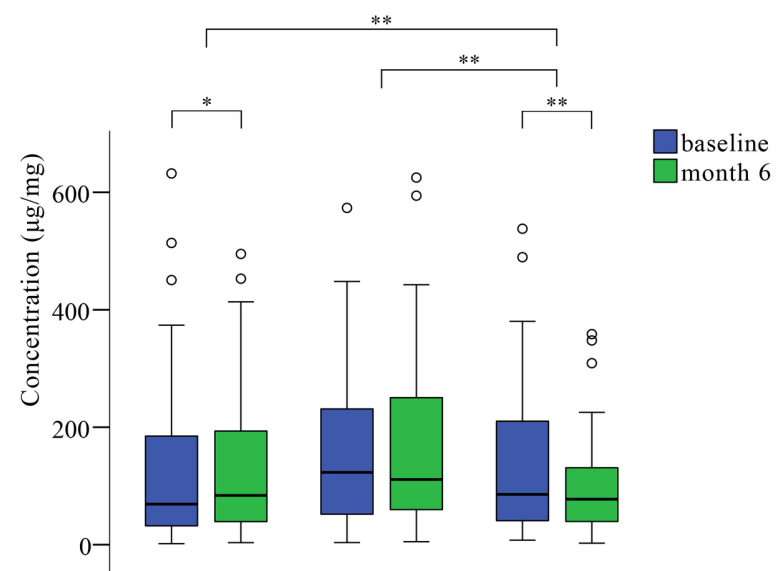

Lower-fat diet Moderate-fat diet Higher-fat diet

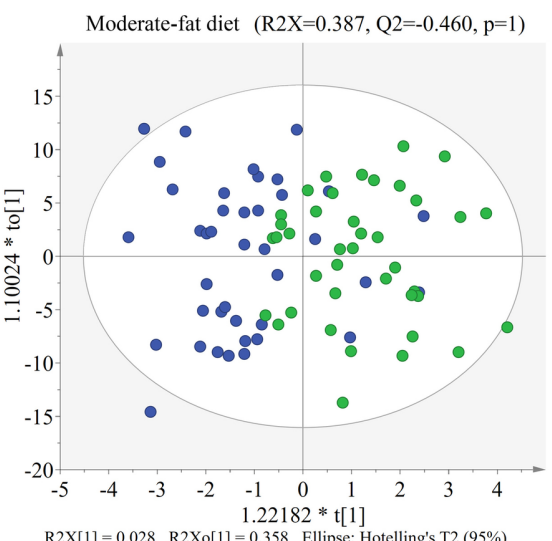

$\mathrm{D}$
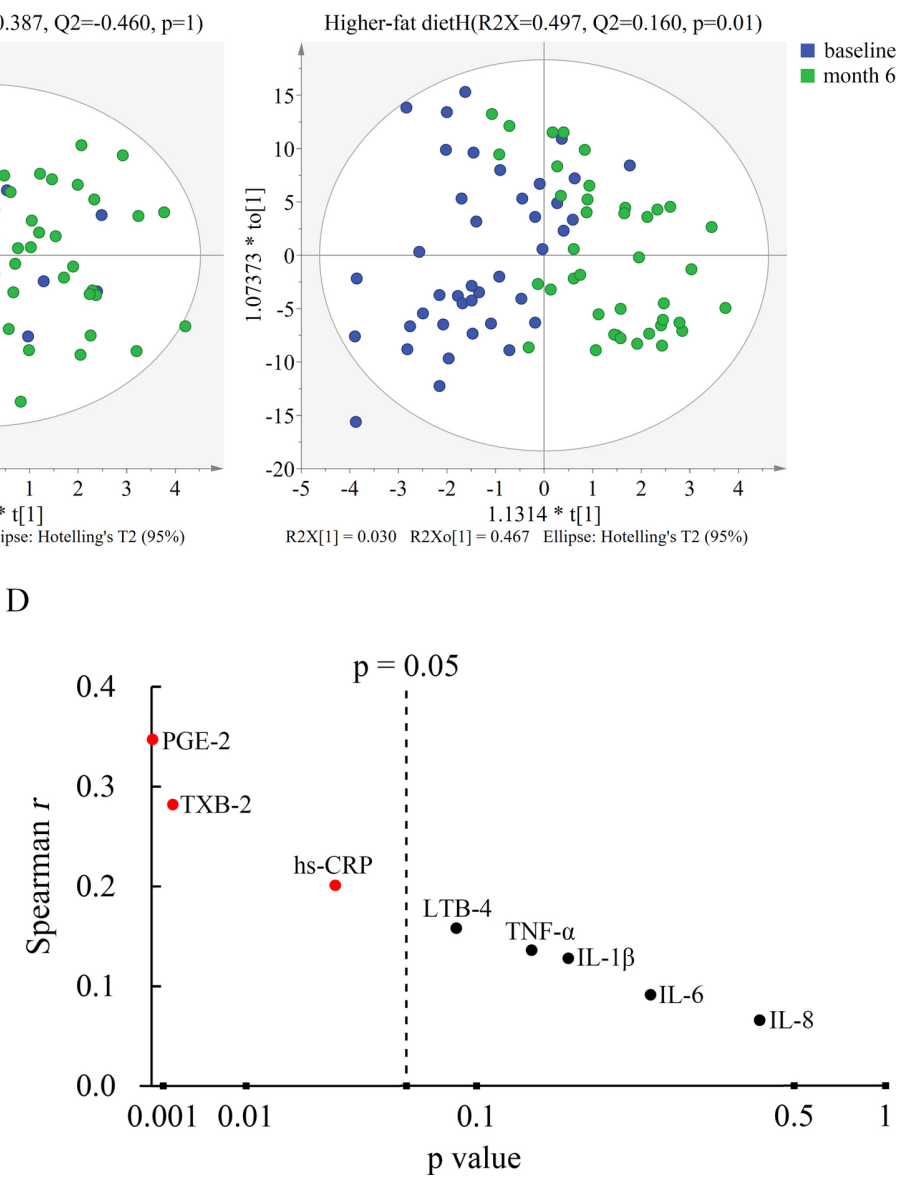

$\mathrm{C}$
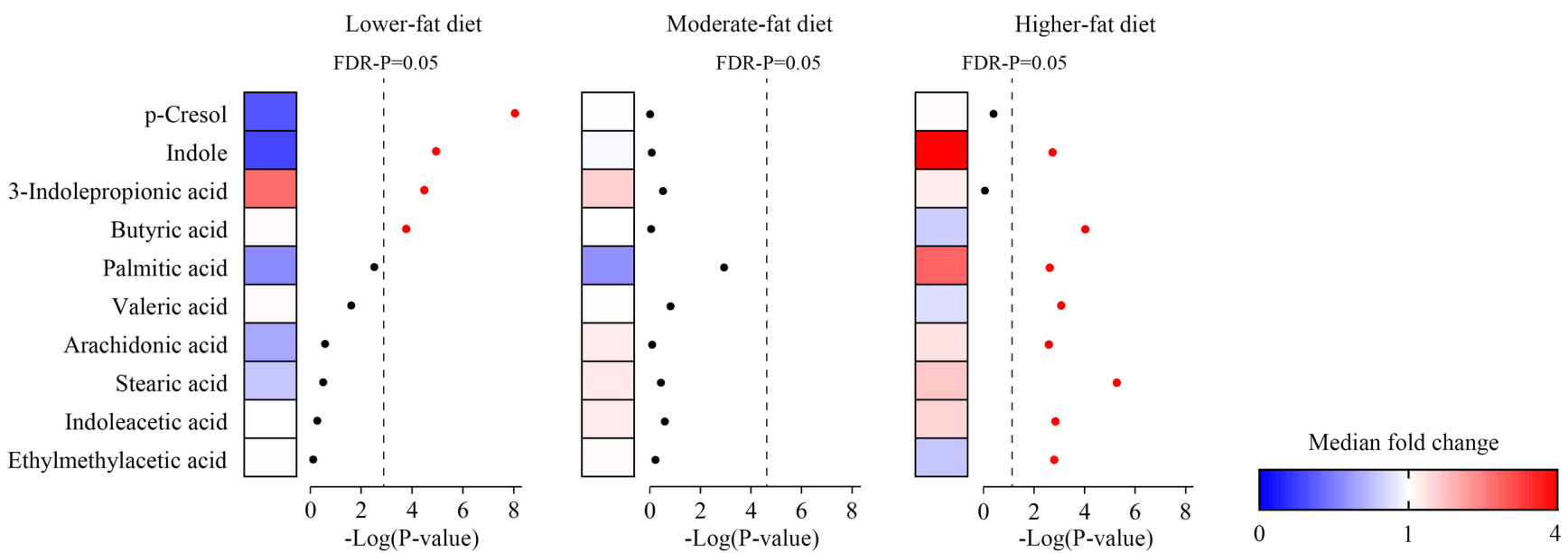

Figure 4 Faecal metabolomics changed after the intervention. (A) OPLS-DA score plots comparing the faecal metabolites before and after the intervention in each group. P values are evaluated by CV-ANOVA. (B) Faecal total short-chain fatty acids concentration before and after the intervention. ${ }^{*} p<0.05$ and ${ }^{* *} p<0.01$. (C) Heatmap of the median fold changes in faecal metabolite concentrations before and after the intervention. (D) Associations between change in faecal arachidonic acid concentration and changes in plasma proinflammatory marker levels. CV-ANOVA, crossvalidation analysis of variance; FDR, false discovery rate; hs-CRP, high sensitivity C-reactive protein; IL, interleukin; LTB ${ }_{4^{\prime}}$ leukotriene $B_{4^{\prime}}$ OPLS-DA, orthogonal projection to latent structure-discriminant analysis; $\mathrm{PGE}_{2^{\prime}}$ prostaglandin $\mathrm{E}_{2:}$ TNF- $\alpha$, tumour necrosis factor $\alpha_{i} \mathrm{TXB}_{2^{\prime}}$, thromboxane $B_{2}$.

Further stratified analysis by metabolite categories showed that amino acid metabolites were significantly altered in the lower-fat group $(\mathrm{p}=0.001)$ and long-chain saturated fatty acids (with 14 or more carbons) were significantly altered in the higher-fat group ( $\mathrm{p}<0.001)$ (online supplementary figure 7). In addition, total short-chain fatty acid (SCFAs) concentrations were significantly decreased in the higher-fat group, compared with other diet groups $(\mathrm{p}<0.001)$ (figure $4 \mathrm{~B})$. Individual metabolite analysis 
identified four significantly changed faecal metabolites, including three amino acid metabolites ( $\mathrm{p}$-cresol, indole and 3-indolepropionic acid) and one SCFA (butyric acid), in the lower-fat group after FDR correction (figure 4C and online supplementary table 4). Among these four metabolites, two were decreased (p-cresol and indole) and two were increased (3-indolepropionic acid and butyric acid). While in the higher-fat group, eight faecal metabolites including long-chain saturated fatty acids, SCFAs and amino acid metabolites were found to be significantly changed. Among them, three were decreased (butyric acid, valeric acid and ethylmethylacetic acid) and five were increased (indole, palmitic acid, stearic acid, arachidonic acid and indoleacetic acid). Compared with the lower-fat group, the higher-fat diet group significantly increased the faecal concentration of palmitic acid, stearic acid, arachidonic acid, indoleacetic acid, indole and p-cresol (all $\mathrm{p}<0.01$ ), while significantly decreasing the faecal concentration of butyric acid, valeric acid and 3-indolepropionic acid (all $\mathrm{p}<0.05$, online supplementary figure 8 ). Since arachidonic acid is the precursor of eicosanoids and other lipid mediators involved in the inflammation process, we specifically tested the correlation between changes in faecal arachidonic acid concentration and changes in plasma proinflammatory markers concentrations. The change in faecal concentration of arachidonic acid was found to be positively associated with the changes in plasma concentrations of $\mathrm{PGE}_{2}(\mathrm{r}=0.35, \mathrm{p}<0.001), \mathrm{TXB}_{2}(\mathrm{r}=0.28, \mathrm{p}=0.002)$ and hs-CRP $(\mathrm{r}=0.20, \mathrm{p}=0.03)$ (figure 4D). Additionally, Spearman correlation analysis showed that the change in Bacteroides was positively associated with the changes in palmitic acid $(\mathrm{r}=0.35, \mathrm{p}=0.004)$, indole $(\mathrm{r}=0.39, \mathrm{p}<0.001)$ and $\mathrm{p}$-cresol $(\mathrm{r}=0.64, \mathrm{p}<0.001)$; the change in Faecalibacterium was positively associated with the change in butyric acid $(\mathrm{r}=0.35, \mathrm{p}=0.004)$; whereas the change in Ruminococcus was negatively associated with the change in p-cresol $(\mathrm{r}=0.32, \mathrm{p}=0.01$, figure 5$)$.

\section{Effects of dietary interventions on inflammatory markers}

Plasma concentration of hs-CRP was significantly increased during the higher-fat diet intervention, compared with the moderate-fat ( $p$ for higher-fat vs moderate-fat $<0.001$ ) and lower-fat ( $p$ for
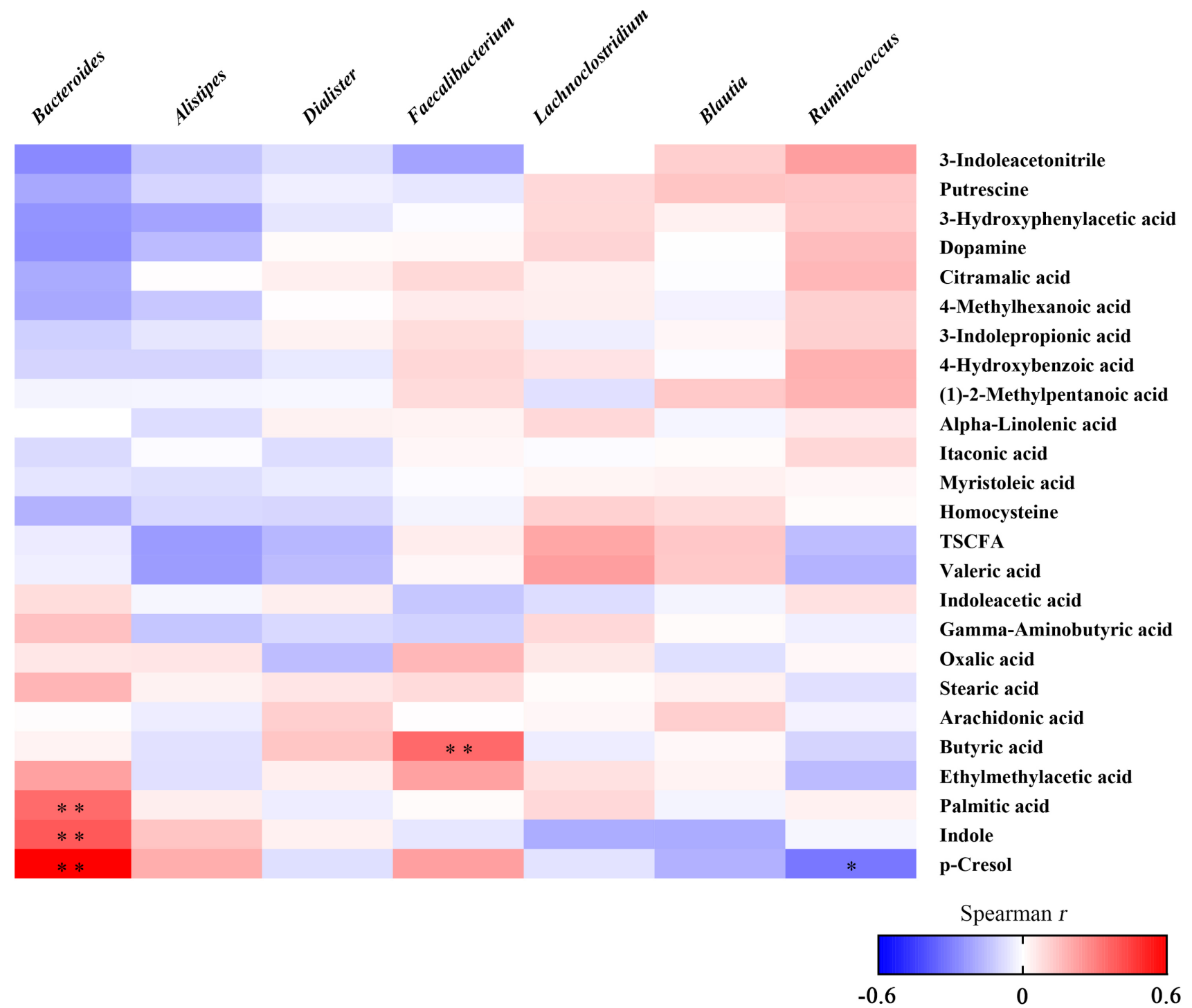

Figure 5 Correlation between changes in faecal metabolites and changes in genus abundance. The heat-map shows the Spearman correlation coefficient between changes in faecal metabolite concentrations and changes in relative abundance of individual genera. The intensity of the colours represents the degree of association between changes in the concentration of faecal metabolites and changes in relative abundance of individual genera as measured by the Spearman's correlations. P values were corrected for multiple testing using the Benjamini-Hochberg false discovery rate. ${ }^{*} p<0.05,{ }^{*} p<0.01$. TSCFA, total short-chain fatty acids. 
Table 2 Effects of dietary interventions on plasma proinflammatory markers $(n=217)$

\begin{tabular}{|c|c|c|c|c|}
\hline $\begin{array}{l}\text { Proinflammatory } \\
\text { factors* }\end{array}$ & $\begin{array}{l}\text { Lower-fat diet } \\
(n=73)\end{array}$ & $\begin{array}{l}\text { Moderate-fat } \\
\text { diet } \\
(n=73)\end{array}$ & $\begin{array}{l}\text { Higher-fat } \\
\text { diet } \\
(n=71)\end{array}$ & $P$ valuet \\
\hline \multicolumn{5}{|l|}{ hs-CRP (mg/L) } \\
\hline baseline & $0.3 \pm 0.6$ & $0.4 \pm 0.4$ & $0.3 \pm 0.4$ & \\
\hline month 6 & $0.2 \pm 0.4$ & $0.3 \pm 0.3$ & $0.4 \pm 0.8$ & \\
\hline change & $-0.1 \pm 0.3^{b}$ & $0.0 \pm 0.3^{b}$ & $0.1 \pm 0.4^{\mathrm{a}}$ & $<0.001$ \\
\hline \multicolumn{5}{|l|}{ IL-6 (pg/mL) } \\
\hline baseline & $29.8 \pm 24.5$ & $34.5 \pm 30.1$ & $30.7 \pm 35.5$ & \\
\hline month 6 & $26.2 \pm 28.2$ & $24.4 \pm 21.0$ & $20.4 \pm 25.9$ & \\
\hline change & $-5.2 \pm 42.1$ & $-7.4 \pm 31.4$ & $-1.1 \pm 49.8$ & 0.62 \\
\hline \multicolumn{5}{|l|}{ IL-8 (pg/mL) } \\
\hline baseline & $52.6 \pm 34.9$ & $52.6 \pm 36.4$ & $55.2 \pm 53.9$ & \\
\hline month 6 & $60.8 \pm 42.1$ & $55.9 \pm 35.8$ & $57.8 \pm 44.8$ & \\
\hline change & $2.4 \pm 54.9$ & $0.0 \pm 40.8$ & $-6.6 \pm 46.7$ & 0.67 \\
\hline \multicolumn{5}{|l|}{ IL-1 $\beta(p g / m L)$} \\
\hline baseline & $2.0 \pm 1.6$ & $1.7 \pm 1.3$ & $1.9 \pm 1.3$ & \\
\hline month 6 & $1.1 \pm 1.3$ & $1.3 \pm 1.2$ & $1.0 \pm 1.2$ & \\
\hline change & $-0.8 \pm 1.7$ & $-0.5 \pm 1.5$ & $-0.8 \pm 1.6$ & 0.73 \\
\hline \multicolumn{5}{|l|}{ TNF- $\alpha$ (pg/mL) } \\
\hline baseline & $53.3 \pm 52.9$ & $62.7 \pm 48.7$ & $66.5 \pm 71.4$ & \\
\hline month 6 & $72.0 \pm 75.1$ & $68.1 \pm 59.6$ & $66.2 \pm 76.4$ & \\
\hline change & $13.3 \pm 65.8$ & $4.9 \pm 66.4$ & $2.8 \pm 84.5$ & 0.55 \\
\hline \multicolumn{5}{|l|}{$\mathrm{LTB}_{4}(\mathrm{pg} / \mathrm{mL})$} \\
\hline baseline & $162.1 \pm 431.0$ & $195.5 \pm 366.7$ & $176.6 \pm 266.4$ & \\
\hline month 6 & $127.4 \pm 166.8$ & $207.6 \pm 331.4$ & $198.4 \pm 184.9$ & \\
\hline change & $-47.2 \pm 128.7^{b}$ & $5.7 \pm 179.0^{\mathrm{a}}$ & $-4.5 \pm 154.9^{\mathrm{a}}$ & 0.003 \\
\hline \multicolumn{5}{|l|}{$\mathrm{TXB}_{2}$ (ng/mL) } \\
\hline baseline & $4.0 \pm 6.3$ & $4.1 \pm 5.5$ & $5.1 \pm 7.9$ & \\
\hline month 6 & $2.7 \pm 5.8$ & $4.1(8.8)$ & $4.4(7.8)$ & \\
\hline change & $-1.1 \pm 2.9^{b}$ & $-0.3 \pm 4.7^{\mathrm{a}}$ & $0.3 \pm 5.7^{a}$ & $<0.001$ \\
\hline \multicolumn{5}{|l|}{$\mathrm{PGE}_{2}(\mathrm{ng} / \mathrm{mL})$} \\
\hline baseline & $1.3 \pm 1.6$ & $1.0 \pm 1.7$ & $1.3 \pm 1.3$ & \\
\hline month 6 & $0.6 \pm 1.2$ & $1.2 \pm 1.8$ & $0.9 \pm 2.3$ & \\
\hline change & $-0.4 \pm 1.0^{b}$ & $-0.2 \pm 0.8^{\mathrm{a}}$ & $0.0 \pm 1.4^{\mathrm{a}}$ & 0.007 \\
\hline
\end{tabular}

*Data are presented as median \pm IQR owing to non-normal distribution. tComparisons among the three groups were conducted using the Kruskal-Wallis test. For significant inflammation markers, all pairwise comparisons were tested using Wilcoxon test and followed by Bonferroni adjustment. Values with different letters in each row differ significantly. The letter 'a' represents the highest value. hs-CRP, high sensitivity C-reactive protein; IL, interleukin; IQR, interquartile range; $\mathrm{LTB}_{4^{\prime}}$ leukotriene $\mathrm{B}_{4^{\prime}} ; \mathrm{PGE}_{2^{\prime}}$, prostaglandin $\mathrm{E}_{2^{\prime}} ; \mathrm{TNF}-\alpha$, tumour necrosis factor- $\alpha ; \mathrm{TXB}_{2^{\prime}}$ thromboxane $B_{2}$.

higher-fat vs lower-fat $<0.001$ ) diet groups (table 2). Likewise, compared with the lower-fat diet, plasma concentration of the inflammatory mediators $\mathrm{TXB}_{2}$ was increased on the higher-fat diet ( $p$ for higher-fat vs lower-fat=0.02). Additionally, $\mathrm{LTB}_{4}$ and $\mathrm{PGE}_{2}$ were significantly decreased in the lower-fat diet group, when compared with moderate-fat diet group ( $\mathrm{p}$ for lower-fat vs moderate-fat $=0.006,0.003$, respectively) and higher-fat diet group ( $\mathrm{p}$ for lower-fat vs higher-fat $=0.02,<0.001$, respectively). However, the changes in proinflammatory cytokines including IL-1 $\beta$, IL-6, IL- 8 and TNF- $\alpha$ showed no significant differences among the three groups after the 6-month diet intervention.

\section{DISCUSSION}

We believe that the present randomised, controlled-feeding trial, is the first study to investigate the effect of different proportions of dietary fat intake on gut microbiota, faecal metabolomic profiles and plasma inflammatory factors in healthy young adults drawn from a population whose diet is in a state of transition. The findings of our study suggest that although the three diets did not induce major changes of the global gut microbiota composition, the higher-fat diet had overall unfavourable effects on several important biomarkers, while the lower-fat diet with a macronutrient distribution corresponding to those consumed in China until some 30 years ago, appeared to have beneficial effects.

Numerous rodent studies have reported that a high-fat diet might appreciably unbalance the gut microbiota composition. ${ }^{21}$ However, the effect of diet on the human gut is complex given the considerable individual variation in the response of the gut microbiota to dietary intervention. ${ }^{22} 23$ Our findings are in keeping with previous studies, which indicate that the overall structure of gut microbiota is principally influenced by long-term dietary intake. ${ }^{10}{ }^{13-15} 2324$ Short-term effects have mainly been reported in studies with small sample size, ${ }^{25} 26$ and characterised by extreme and unrealistic dietary intakes ${ }^{25}$ or when the subjects studied were at high cardiometabolic risk. ${ }^{27-29}$ A 10-day clinical trial with nine participants reported that the composition and function of the microbiota were rapidly changed when carbohydrates were eliminated from the diet, a dietary intervention which is neither sustainable nor desirable in the long term. ${ }^{25}$ In much more moderate dietary interventions that can be sustained in humans, high interindividual variations among subjects might have masked minor changes in distinct taxonomic groups. ${ }^{30} \mathrm{It}$ has been reported that diet might have a greater influence on the metabolic activity of the gut microbiota than on its taxonomic composition. ${ }^{16}$ In accordance with this proposition, in our study diets differing in dietary fat content provoked a marked shift of faecal concentrations of metabolites produced by the gut microbiota.

We found Blautia and Faecalibacterium, genera known to contain butyrate-producing bacteria, were increased in the lower-fat diet group. Blautia is a group of bacteria containing various acetate and butyrate producers and reported to have a lower relative abundance in patients with T2D than in healthy controls. ${ }^{31} 32$ Faecalibacterium contains anti-inflammatory and functionally important bacteria. ${ }^{33}$ Higher abundance of $F$. prausnitzii was associated with a reduction of systematic low-grade inflammation among obese subjects receiving bariatric surgery. ${ }^{34}$ Many previous studies have reported the benefits of dietary fibre on gut microbiota. ${ }^{35}$ In our study, the amount of carbohydrate was highest in the lower-fat diet group, mainly from white rice and wheat flour (bread). The proportion of resistant starch has been reported to be $3 \%$ in white rice and $2 \%$ in bread. ${ }^{3637}$ Given that dietary fibre intake did not differ among the three groups, it is possible that the favourable effects of the lower-fat diet in our study might be due to the increased amount of resistant starch which can, like dietary fibre, be fermented by the gut microbiota, with associated health benefits.

In contrast, the abundance of Faecalibacterium was decreased and Bacteroides and Alistipes were increased after the higher-fat diet intervention. Among the Chinese population, Bacteroides and Alistipes were found to be more abundant in patients with T2D than in subjects with normal glucose metabolism. ${ }^{38}$ These changes at genus level indicated that dietary fat content had a selective effect on the human gut microbiota, which might have clinical significance among healthy young adults. The higher-fat diet induced a reduction in faecal butyrate acid and SCFAs as opposed to the lower-fat diet group. This is probably due to the reduction in dietary carbohydrate intake. ${ }^{39}$ It has been suggested that the benefits of 
a 'healthy diet' are mediated via the anti-inflammatory effects of SCFAs and other bioactive compounds produced by the gut microbiota. ${ }^{40}$ Several observations in our study pointed towards an effect of gut microbiota-mediated proinflammatory factors. Among the individual genera which responded to the dietary intervention, the abundance of Faecalibacterium was directly related to the concentration of butyric acid, as suggested by the positive association between changes in butyrate concentration and the abundance of Faecalibacterium. A study using faecal microbiota transplantation from lean donors to insulin-resistant patients with metabolic syndrome demonstrated that faeces from lean subjects were associated with enhanced numbers of butyrate-producing bacteria and increased insulin sensitivity. ${ }^{41}$ In contrast, a significant reduction of dietary carbohydrates in omnivores led to much lower levels of faecal SCFAs and decreased bacterial numbers. ${ }^{39}$ The protective role of SCFAs against different types of disease is well documented; in particular, butyric acid can serve as the energy substrate for epithelial cells of the gut and confer anti-inflammatory effects. ${ }^{42}$ The reduction in SCFAs induced by the higher-fat diet was presumably detrimental for metabolic health.

In addition to the reduction of SCFAs, the higher-fat diet induced significant alterations in long-chain fatty acids metabolism. The faecal concentrations of palmitic acid (C16:0) and stearic acid (C18:0) were significantly increased in the higher-fat group. Palmitic acid and stearic acid, the main saturated fatty acids, in food and tissues, have been considered to stimulate inflammatory signalling in macrophages, adipocytes, myocytes, hepatocytes, etc. ${ }^{43}$ Epidemiological studies showed that plasma phospholipid palmitic acid and stearic acid were positively associated with the incidence of T2D and cardiovascular diseases. ${ }^{44}$ Notably, the predicted lipopolysaccharide biosynthesis and arachidonic acid metabolism pathways were also increased in response to the higher-fat diet. Lipopolysaccharide is known to induce the release of arachidonic acid and its inflammation-involving metabolites, such as prostaglandins, thromboxane and leukotrienes. ${ }^{45}$ It should be noted that the intake of polyunsaturated fatty acids (PUFAs) was relatively high in the higher-fat diet group ( $24 \%$ of total energy) owing to exclusive use of soybean oil, which is rich in n-6 PUFA. A higher intake of $n-6$ PUFA has been reported to have proinflammatory effects. ${ }^{46}$ Consistent with the predicted pathway analysis, we found the faecal concentration of arachidonic acid (the precursor of proinflammatory compounds like $\mathrm{PGE}_{2}, \mathrm{TXB}_{2}$ and $\mathrm{LTB}_{4}$ ) was indeed increased in the higher-fat group. The positive association between changes in faecal arachidonic acid concentration and the changes in plasma levels of inflammatory makersnamely, $\mathrm{PGE}_{2}$ and $\mathrm{TXB}_{2}$, suggested a potential mechanism through which the amount of dietary fat consumption affects inflammation via gut microbiota.

Interestingly, although the same amount and quality of protein was provided in each diet group, we found that the lower-fat diet group induced a significantly alteration in the amino acids metabolism, indicating enhanced protein degradation by the gut microbiota. The results reinforced the view that luminal substrate availability is also an important variable for fixing bacterial metabolite concentration. ${ }^{16}$ The lower-fat diet group induced reduction of faecal concentrations of indole and p-cresol, the host microbiota cometabolites derived from amino acids, while the higher-fat diet led to an increase in indole concentration. Indole is the precursor of indoxyl sulfate, which has been linked with hypertension and cardiovascular disease in people with chronic kidney disease. ${ }^{47}$ Likewise, $\mathrm{p}$-cresol, the precursor of p-cresol sulfate, has been shown to act as an inhibitor of colonocyte oxygen consumption and as a genotoxic agent in vitro. ${ }^{48}$ Production rates of these potentially toxic compounds are found to be markedly lower in vegetarians than in people consuming an unrestricted diet. ${ }^{49}$ It has also been reported that bacterial diversity is higher among populations adopting a lower-fat diet like a vegetarian diet and can be modified through dietary intervention in obese subjects. ${ }^{50}$ It should be noted that the microbial $\alpha$-diversity (indicated by the Shannon index) was increased in the lower-fat group compared with the higher-fat diet group. Increased diversity has been associated with improved health in the elderly, and reduction of gut microbial diversity has been linked to increased risk of gastrointestinal diseases and proinflammatory characteristics. ${ }^{14} 51$ Thus, in addition to increased SCFAs, our data suggest that the lower-fat diet can decrease the luminal concentration of these deleterious compounds while increasing the gut microbial $\alpha$-diversity.

Correlation analysis allowed us to identify several new bacterial genera potentially implicated in the host metabolic health. We observed negative associations of Blautia abundance with serum concentration of TC, LDL-C and non-HDL-C, whereas the opposite was seen for Bacteroides, a genus that we found to be increased after higher-fat consumption. A previous clinical trial reported that T2D and hyperlipidaemia patients were ameliorated by metformin and a herbal formula with increased abundance of Bautia, which was correlated significantly with the improvement in circulating glucose and lipids. ${ }^{52}$ Also, in line with a clinical trial in which Blautia was found to be negatively associated with TC and non-HDL-C, we observed similar negative associations between Blautia and blood lipid profiles. ${ }^{53}$ The enrichment of Blautia in the lower-fat group suggested that the diet-induced change in gut microbiota might be related to the host lipid homoeostasis in healthy young adults and possibly, be a future target for the management of subjects with cardiometabolic diseases.

A limitation of this study was that faecal sampling was carried out only at baseline and at the end of the trial. A more complete picture of changes in gut microbiota would have been possible with more frequent sampling. In addition, all three groups lost weight and weight loss differed among groups after dietary intervention. Whether weight loss led to the changes in gut microbiota and faecal metabolomics or vice versa needs to be studied further. Another limitation relates to the generalisability of our study since our subjects were healthy young non-obese adults; the results might not apply to people at high risk of cardiometabolic diseases. The strengths of this study included the controlled-feeding design, the large sample size and the relatively long intervention period. In contrast to previous studies, we applied 16S rRNA amplicon profiling and quantitatively targeted bacterial metabolomics, which allowed us to understand both gut microbiota response and bacterial metabolites to gain more information about host-gut microbiota metabolic interactions in response to dietary fat content.

\section{CONCLUSION}

In summary, compared with a lower-fat diet, long-term consumption of a higher-fat diet appears to be undesirable owing to changes in gut microbiota, faecal metabolomic profiles and proinflammatory factors for healthy young adults whose diet is in transition from the traditionally consumed lower fat, higher carbohydrate diet to one characterised by an appreciably higher fat content. These findings might also have relevance in developed countries in which fat intake is already high.

\section{Author affiliations}

Department of Food Science and Nutrition, Zhejiang University, Hangzhou, China 2Department of Nutrition, Harvard T. H. Chan School of Public Health, Boston, USA 
${ }^{3}$ No. 1 Department of Nutrition, Chinese People's Liberation Army General Hospital, Beijing, China

${ }^{4}$ Department of Gastroenterology, School of Medicine, Zhejiang University, Hangzhou, China

${ }^{5}$ Department of Epidemiology and Biostatistics, School of Public Health, Peking University, Beijing, China

${ }^{6}$ Institute of Basic Medical Science, Westlake University, Hangzhou, China

${ }^{7}$ Department of Nutrition, Dietetics and Food, Monash University, Melbourne,

Australia

${ }^{8}$ Department of Human Nutrition and Medicine, University of Otago, Otago, New Zealand

${ }^{9}$ Institute of Nutrition and Health, Qingdao University, Qingdao, China

Acknowledgements We thank all the participants, the investigators, on-site staff, kitchen staff and all members of the Duo Li Laboratory for participating in scientific discussions about the manuscript.

Contributors Conception and design of the study: DL, YW, and FW. Collection of data: JL, DJ, JY, JiZ, HL and RW. Analysis and interpretation of data: YW, FW, JT, TH and JuZ. Drafting of the manuscript: YW and FW. Critical revision of the manuscript for important intellectual content: AJS, JM, and DL. Administrative support and study supervision: DL and JY. All authors read, revised and approved the final draft.

Funding This study was funded by the National Basic Research Program of China (2015CB553604) and China Postdoctoral Science Foundation (2018M642466). The funders of the study had no role in study design, data collection, data analysis, data interpretation, or writing of the manuscript.

Competing interests None declared.

Patient consent Obtained.

Ethics approval Institutional review board.

Provenance and peer review Not commissioned; externally peer reviewed.

\section{REFERENCES}

1 Austin GL, Ogden LG, Hill JO. Trends in carbohydrate, fat, and protein intakes and association with energy intake in normal-weight, overweight, and obese individuals: 1971-2006. Am J Clin Nutr 2011;93:836-43.

2 Livesey G, Taylor R, Livesey H, et al. Is there a dose-response relation of dietary glycemic load to risk of type 2 diabetes? Meta-analysis of prospective cohort studies. Am J Clin Nutr 2013;97:584-96.

3 Te Morenga L, Mallard S, Mann J. Dietary sugars and body weight: systematic review and meta-analyses of randomised controlled trials and cohort studies. BMJ 2012;346:e7492.

4 Du SF, Wang HJ, Zhang B, et al. China in the period of transition from scarcity and extensive undernutrition to emerging nutrition-related non-communicable diseases, 1949-1992. Obes Rev 2014;15(Suppl 1):8-15.

5 Zhai FY, Du SF, Wang ZH, et al. Dynamics of the Chinese diet and the role of urbanicity, 1991-2011. Obesity Reviews 2014;15(Suppl. 1):16-26.

6 Wan Y, Wang F, Yuan J, et al. Effects of macronutrient distribution on weight and related cardiometabolic profile in healthy non-obese chinese: a 6-month, randomized controlled-feeding trial. EBioMedicine 2017;22:200-7.

7 Clemente JC, Ursell LK, Parfrey LW, et al. The impact of the gut microbiota on human health: an integrative view. Cell 2012;148:1258-70.

8 Turnbaugh PJ, Ridaura VK, Faith JJ, et al. The effect of diet on the human gut microbiome: a metagenomic analysis in humanized gnotobiotic mice. Sci Trans/ Med 2009:1:6ra14-14r.

9 Serino M, Luche E, Gres S, et al. Metabolic adaptation to a high-fat diet is associated with a change in the gut microbiota. Gut 2012;61:543-53.

10 De Filippo C, Cavalieri D, Di Paola M, et al. Impact of diet in shaping gut microbiota revealed by a comparative study in children from Europe and rural Africa. Proc Natl Acad Sci U S A 2010;107:14691-6.

11 Ou J, Carbonero F, Zoetendal EG, et al. Diet, microbiota, and microbial metabolites in colon cancer risk in rural Africans and African Americans. Am J Clin Nutr 2013;98:111-20.

12 Ley RE, Turnbaugh PJ, Klein S, et al. Microbial ecology: human gut microbes associated with obesity. Nature 2006;444:1022-3.

13 Wu GD, Chen J, Hoffmann C, et al. Linking long-term dietary patterns with gut microbial enterotypes. Science 2011;334:105-8.

14 Claesson MJ, Jeffery IB, Conde S, et al. Gut microbiota composition correlates with diet and health in the elderly. Nature 2012;488:178-84.

15 Cotillard A, Kennedy SP, Kong LC, et al. Dietary intervention impact on gut microbial gene richness. Nature 2013:500:585-8.

16 Wu GD, Compher C, Chen EZ, et al. Comparative metabolomics in vegans and omnivores reveal constraints on diet-dependent gut microbiota metabolite production. Gut 2016;65:63-72.

17 Wan Y, Wang F, Yuan J, et al. Optimal dietary macronutrient distribution in China (ODMDC): a randomised controlled-feeding trial protocol. Asia Pac J Clin Nutr 2017;26:972-80.
18 Zhao L, Ni Y, Su M, et al. High throughput and quantitative measurement of microbial metabolome by gas chromatography/mass spectrometry using automated alkyl chloroformate derivatization. Anal Chem 2017;89:5565-77.

19 Schloss PD, Westcott SL, Ryabin T, et al. Introducing mothur: open-source, platformindependent, community-supported software for describing and comparing microbial communities. Appl Environ Microbiol 2009;75:7537-41.

20 Arumugam M, Raes J, Pelletier E, et al. Enterotypes of the human gut microbiome. Nature 2011:473:174-80.

21 Murphy EA, Velazquez KT, Herbert KM. Influence of high-fat diet on gut microbiota: a driving force for chronic disease risk. Curr Opin Clin Nutr Metab Care 2015; 18:515-20.

22 Walker AW, Ince J, Duncan SH, et al. Dominant and diet-responsive groups of bacteria within the human colonic microbiota. Isme J 2011;5:220-30.

23 Salonen A, Lahti L, Salojärvi J, et al. Impact of diet and individual variation on intestinal microbiota composition and fermentation products in obese men. Isme J 2014;8:2218-30.

24 Roager HM, Vogt JK, Kristensen M, et al. Whole grain-rich diet reduces body weight and systemic low-grade inflammation without inducing major changes of the gut microbiome: a randomised cross-over trial. Gut 2019;68:83-93.

25 David LA, Maurice CF, Carmody RN, et al. Diet rapidly and reproducibly alters the human gut microbiome. Nature 2014;505:559-63.

26 Whisner $\mathrm{CM}$, Martin BR, Nakatsu CH, et al. Soluble corn fiber increases calcium absorption associated with shifts in the gut microbiome: a randomized dose-response trial in free-living pubertal females. J Nutr 2016;146:1298-306.

27 Xiao S, Fei N, Pang X, et al. A gut microbiota-targeted dietary intervention for amelioration of chronic inflammation underlying metabolic syndrome. FEMS Microbiol Ecol 2014;87:357-67.

28 Zhang C, Yin A, Li H, et al. Dietary modulation of gut microbiota contributes to alleviation of both genetic and simple obesity in children. EBioMedicine 2015;2:968-84.

$29 \mathrm{Xu}$ J, Lian F, Zhao L, et al. Structural modulation of gut microbiota during alleviation of type 2 diabetes with a Chinese herbal formula. Isme J 2015:9:552-62.

30 Flint $\mathrm{HJ}$, Scott KP, Louis $\mathrm{P}$, et al. The role of the gut microbiota in nutrition and health. Nat Rev Gastroenterol Hepatol 2012;9:577-89.

31 Park SK, Kim MS, Bae JW. Blautia faecis sp. nov., isolated from human faeces. Int J Syst Evol Microbiol 2013;63:599-603.

32 Inoue R, Ohue-Kitano R, Tsukahara T, et al. Prediction of functional profiles of gut microbiota from 165 rRNA metagenomic data provides a more robust evaluation of gut dysbiosis occurring in Japanese type 2 diabetic patients. J Clin Biochem Nutr 2017;61:217-21.

33 Pryde SE, Duncan SH, Hold GL, et al. The microbiology of butyrate formation in the human colon. FEMS Microbiol Lett 2002;217:133-9.

34 Furet JP, Kong LC, Tap J, et al. Differential adaptation of human gut microbiota to bariatric surgery-induced weight loss: links with metabolic and low-grade inflammation markers. Diabetes 2010;59:3049-57.

35 Makki K, Deehan EC, Walter J, et al. The impact of dietary fiber on gut microbiota in host health and disease. Cell Host Microbe 2018;23:705-15.

36 Yang $\mathrm{CZ}$, Shu XL, Zhang LL, et al. Starch properties of mutant rice high in resistant starch. J Agric Food Chem 2006;54:523-8.

37 Sajilata MG, Singhal RS, Kulkarni PR. Resistant starch? A review. Compr Rev Food Sci Food Saf 2006;5:1-17.

38 Qin J, Li Y, Cai Z, et al. A metagenome-wide association study of gut microbiota in type 2 diabetes. Nature 2012;490:55-60.

39 Duncan SH, Belenguer A, Holtrop G, et al. Reduced dietary intake of carbohydrates by obese subjects results in decreased concentrations of butyrate and butyrate-producing bacteria in feces. Appl Environ Microbiol 2007;73:1073-8.

40 Fardet A. New hypotheses for the health-protective mechanisms of whole-grain cereals: what is beyond fibre? Nutr Res Rev 2010;23:65-134.

41 Udayappan SD, Hartstra AV, Dallinga-Thie GM, et al. Intestinal microbiota and faecal transplantation as treatment modality for insulin resistance and type 2 diabetes mellitus. Clin Exp Immunol 2014;177:24-9.

42 Ríos-Covián D, Ruas-Madiedo P, Margolles A, et al. Intestinal short chain fatty acids and their link with diet and human health. Front Microbiol 2016:7:185.

43 Nguyen MT, Favelyukis S, Nguyen AK, et al. A subpopulation of macrophages infiltrates hypertrophic adipose tissue and is activated by free fatty acids via Toll-like receptors 2 and 4 and JNK-dependent pathways. J Biol Chem 2007;282:35279-92.

44 Fretts AM, Mozaffarian D, Siscovick DS, et al. Plasma phospholipid saturated fatty acids and incident atrial fibrillation: the Cardiovascular Health Study. J Am Heart Assoc 2014:3:e889.

45 Shoeb M, Yadav UC, Srivastava SK, et al. Inhibition of aldose reductase prevents endotoxin-induced inflammation by regulating the arachidonic acid pathway in murine macrophages. Free Radic Biol Med 2011;51:1686-96.

46 Patterson E, Wall R, Fitzgerald GF, et al. Health implications of high dietary omega-6 polyunsaturated Fatty acids. J Nutr Metab 2012;2012:1-16.

47 Barreto FC, Barreto DV, Liabeuf S, et al. Serum indoxyl sulfate is associated with vascular disease and mortality in chronic kidney disease patients. Clin J Am Soc Nephrol 2009;4:1551-8. 
48 Andriamihaja M, Lan A, Beaumont M, et al. The deleterious metabolic and genotoxic effects of the bacterial metabolite p-cresol on colonic epithelial cells. Free Radic Biol Med 2015;85:219-27.

49 Patel KP, Luo FJ, Plummer NS, et al. The production of $p$-cresol sulfate and indoxyl sulfate in vegetarians versus omnivores. Clin J Am Soc Nephrol 2012;7:982-8

50 do Rosario VA, Fernandes R, Trindade EB. Vegetarian diets and gut microbiota: important shifts in markers of metabolism and cardiovascular disease. Nutr Rev 2016;74:444-54.
51 Ott SJ, Musfeldt M, Wenderoth DF, et al. Reduction in diversity of the colonic mucosa associated bacterial microflora in patients with active inflammatory bowel disease. Gut 2004;53:685-93.

52 Tong $\mathrm{X}$, Xu J, Lian F, et al. Structural alteration of gut microbiota during the amelioration of human type 2 diabetes with hyperlipidemia by metformin and a traditional chinese herbal formula: a multicenter, randomized, open label clinical trial. MBio 2018;9:e2317-92.

53 Upadhyaya B, McCormack L, Fardin-Kia AR, et al. Impact of dietary resistant starch type 4 on human gut microbiota and immunometabolic functions. Sci Rep 2016;6:28797 